\title{
R-spodin2 enhances canonical Wnt signaling to maintain the stemness of glioblastoma cells
}

\author{
Si Liu ${ }^{1}$, Kin Pong $U^{1,2}$, Jieting Zhang ${ }^{1,2}$, Lai Ling Tsang ${ }^{1,2}$, Jiawei Huang ${ }^{1}$, Shui Ping Tu ${ }^{3}$ and Xiaohua Jiang ${ }^{1,2^{*}} \mathbb{E}$
}

\begin{abstract}
Background: As newly identified Wnt enhancer, R-spondin gene family members have been linked to various cancers; however, their role in isocitrate dehydrogenase-wildtype subtype of human glioblastoma (GBM) cells remains unknown.

Methods: Human U87 and U251 cell lines were used to perform the experiments. GBM stem-like cells were enriched in stem cell growth media and induced to differentiate using retinoid acid or growth factor deprivation. Wnt ${ }^{\text {high }}$ and Wnt ${ }^{\text {low }}$ subpopulations were isolated and evaluated by MTS, sphere formation, transwell migration and xenograft formation assays.

Results: R-spondin 2 but not R-spondin 3 potentiates Wnt/ $\beta$-catenin signaling in GBM cell lines. While R-spondin 2 does not affect cell growth, it induces the expression of pluripotent stem cell markers in combination with Wnt3A. GBM stem-like cells are endowed with intrinsic high activity of $\beta$-catenin signaling, which can be further intensified by R-spondin 2. In addition, R-spondin2 promotes stem cell self-renewal and suppresses retinoid acid- or growth factor deprivation-induced differentiation, indicating R-spondin 2 maintains stem cell traits in GBM. On the other hand, we identify subpopulations of GBM cells that show distinctive responsiveness to Wnt/ $\beta$-catenin signaling. Interestingly, Wnt ${ }^{\text {high }}$ and Wnt ${ }^{\text {low }}$ cells display distinctive biologic properties. Moreover, Wnt ${ }^{\text {high }}$ cell-inoculated xenografts exhibit enhanced tumorigenicity and increased expression levels of R-spondin 2 compared to Wnt ${ }^{\text {low }}$ cell-inoculated xenografts.
\end{abstract}

Conclusion: Our study reveals a novel regulatory mechanisms underlying the over-activation of $\beta$-catenin-mediated signaling in the pathogenesis of GBM.

Keywords: R-spondin2, Glioblastoma, Stemness, Wnt, Cancer stem cells

\section{Background}

Glioblastoma multiforme (GBM), one of the most devastating and lethal forms of human cancer, has a median survival rate of only about 15 months [1]. In recent years, so-called cancer stem cells (CSCs) have been isolated from human tumors [2]. These cells normally constitute a minority population and are proposed to be the cells

\footnotetext{
*Correspondence: xjiang@cuhk.edu.hk

${ }^{1}$ Key Laboratory for Regenerative Medicine of the Ministry of Education of China, School of Biomedical Sciences, Faculty of Medicine, The Chinese University of Hong Kong, Hong Kong, SAR, People's Republic of China Full list of author information is available at the end of the article
}

from which tumors are derived and maintained. The corollary of the existence of CSCs is that tumors are not homogeneous but rather, are comprised of a heterogeneous assortment of abnormally differentiated cells derived from an abnormal clonal stem cell pool [3]. The glioblastoma stem cells (GSCs) have been identified in GBM and are likely responsible for the failure of treatment and high recurrence rates [4]. GSCs are capable of self-renewal and differentiation, and de novo tumor formation when implanted in xenograft models $[5,6]$. Furthermore, GSCs possess unique surface markers, such as CD133, SSEA1 , Nestin and OLIG2 [5, 7-9], some of which modulate 
characteristic signaling pathways and play key roles in GBM vascular formation [10, 11].

It has been well established that $\mathrm{Wnt} / \beta$-catenin pathway contributes to cancer development since the discovery that Wnt1 was capable of promoting breast cancer 30 years ago [12]. This recognition has been largely strengthened by the findings that intracellular components of the $\mathrm{Wnt} / \beta$-catenin signaling cascade such as $A P C$, Axin, and $\beta$-Catenin (CTNNB1), are mutated in a large variety of human cancers [13]. Besides the numerous mutations found in the pathway components, the contribution of various family members of Wnt ligand has also been recognized in human cancers [14]. Notably, therapeutic agents, which target the binding process of Wnt ligands, have been shown to suppress tumor growth in various xenograft models [15-19]. For instance, OMP18R5 (vantictumab), an antibody that blocks Wnt binding to 5 out of the 10 human Frizzled receptors, inhibits the growth of a wide range of human cancers [17], indicating that extracellular Wnt signals play an essential role in cancer development. Compared to other cancers, the role of Wnt/ $\beta$-catenin signaling in GBM is less clear. While aberrant activation of $\mathrm{Wnt} / \beta$-catenin signaling has been involved in the pathogenesis of GBM $[20,21]$ and $\beta$-catenin is a predictive marker of short survival in GBM patients [22], most GBM do not harbor driver mutations in the canonical Wnt signaling pathway. Thus, it is conceivable that other regulatory mechanisms that trigger the over-activation of $\beta$-catenin-mediated signaling are involved in the pathogenesis of GBM [23, 24].

The R-spondin (Rspos) thrombospondin type 1 repeat (TSR1)-containing protein superfamily contains four secreted proteins Rspo1-4 [25], which are emerged as important stroma-derived growth factors driving the renewal of epithelial stem cells in many adult vertebrate tissues [26]. Lgr4-6 are selectively expressed in various tissue stem cells [27] and are the primary high-affinity receptors for Rspos [28]. Mechanistically, Rspos markedly amplify target cell sensitivity to Wnt ligands by neutralizing two transmembrane E3 ubiquitin ligases, Znrf3 and Rnf43, which reduce cell-surface levels of Wnt receptors from internalization and degradation $[29,30]$. Of note, Rspos have been emerging as important regulators of cancer development in recent years. Rspo 2 and Rspo3 were firstly identified as sites of integration for MMTVinduced mammary tumors in mice, which suggest a role of Rspos as breast cancer oncogenes [31]. Subsequently, genomic rearrangements that result in elevated RSPO expression have been identified in human colon cancers and demonstrated to activate Wnt signaling and tumorigenesis [32,33]. More interestingly, it was shown recently that Rspos promoted CSC traits [34], whereas therapeutic targeting on Rspos induced stem cell differentiation
[35, 36]. However, it should be noted that contradictory findings have also been reported by various studies. In colon cancer, R-spondin 1 and R-spondin 2 have been demonstrated to suppress CRC tumorigenesis and progression via Wnt-dependent or-independent mechanisms [37, 38]. Taken together, while Rspos have been identified as Wnt enhancers and implicated in cancer development, the exact role of Rspos in cancer development is still controversial.

Given that aberrant activation of $\mathrm{Wnt} / \beta$-catenin pathway has been implicated in GBM development, we hypothesized that Rspos might modify canonical Wnt signaling in GBM cells, and be indicative of cancer stemness trait regulation. We undertook the present study in two different human isocitrate dehydrogenase (IDH)-wildtype subtype glioblastoma cell lines, U251 and U87, to determine the role of Rspo family members in GBM.

\section{Materials and methods}

\section{GBM cell culture and reagents}

Both of U87 and U251 cell lines are IDH-wildtype subtype of glioblastoma (GBM-IDH-wt) according to the recent change in classification of gliomas. U87 and U251 were purchased from ATCC (Manassas, VA, USA). The authentication of the U251 and U87 cell lines have been tested by short tandem repeat (str) profiling by Department of Pathology, the Chinese University of Hong Kong in 2016 and 2017. Cells were cultured in DMEM medium (Gibco Invitrogen, Grand Island, New York, USA) supplemented with $10 \%$ fetal bovine serum (Gibco) and $1 \%$ penicillin-streptomycin, at $37{ }^{\circ} \mathrm{C}$ and $5 \% \mathrm{CO}_{2}$. Cells were maintained in T75 flasks or T25 flasks, and passaged every $2-3$ days when reached $70-80 \%$ confluence. Recombinant Murine Wnt3A, human R-spondin 2 and R-spondin 3 were purchased from PeproTech (Rocky Hill, NJ).

\section{GSC culture}

The following GSC culture medium was applied to enrich GSCs: DMEM/F12 medium (Thermo Fisher Scientific, Grand Island, NY) supplemented with $1 \times \mathrm{B}-27^{\mathrm{TM}}$ Supplement, serum free (Gibco, 17504044), $20 \mathrm{ng} / \mathrm{ml}$ AnimalFree Recombinant Human EGF (PeproTech, AF-100-15), 20 ng/ml Recombinant Human FGF-basic (154 a.a.) (PeproTech, 100-18B), and $1 \times$ penicillin-streptomycin. Cells were seeded in $\operatorname{Costar}^{\circledR} 24$ Well Clear Flat Bottom Ultra Low Attachment plates (Corning, NY) at 1000 cells $/ \mathrm{ml}$. Spheres were carefully aspired into $15 \mathrm{ml}$ tube, and spin down at $1000 \mathrm{rpm}$ for $3 \mathrm{~min}$. Subsequently, $1 \mathrm{ml}$ warm trypsin was used to digest spheres at room temperature for $3 \mathrm{~min}$. The reaction was stopped by adding $10 \mathrm{ml}$ 
GSC culture media, then single cells were spin down and re-plated.

\section{Lentiviral transduction}

7TGP vector was purchased from Addgene (\#24305). This is a lentiviral GFP-coupled Wnt reporter construct constrains a GFP gene under the control of 7 TCF responsive element, which yields expression of GFP only in cells with activated $\mathrm{Wnt} / \beta$-catenin signaling (Additional file 1: Fig S1C). In brief, $5 \times 10^{6} 293 \mathrm{~T}$ cells were seeded in $10-\mathrm{cm}$ dishes 1 day before transfection. For each dish, $8 \mu \mathrm{g}$ of 7TGP lentiviral vector were mixed with $3 \mu \mathrm{g}$ of the VSV-G envelope plasmid (pMD2.VSVG) and $6 \mu \mathrm{g}$ of the packaging plasmid (pCMVDR8.74). The solution was topped up to $250 \mu \mathrm{l}$ with water and mixed with $250 \mu \mathrm{l} 0.5 \mathrm{M} \mathrm{CaCl}_{2}$. The precipitate was formed by adding $500 \mu \mathrm{l}$ of $2 \times$ HEPES-buffered saline $(280 \mathrm{mM} \mathrm{NaCl}$, $10 \mathrm{mM} \mathrm{KCl}, 1.5 \mathrm{mM} \mathrm{Na}_{2} \mathrm{HPO}_{4}, 12 \mathrm{mM}$ dextrose, $50 \mathrm{mM}$ HEPES, pH7.2) drop-wise while vortexing and added directly to the cells. The medium was replaced after $16 \mathrm{~h}$ and conditioned twice for $24 \mathrm{~h}$.

\section{Analysis of Wnt/ $\beta$-catenin activity and isolation of Wnt ${ }^{\text {high }}$ and Wnt ${ }^{\text {low }}$ cell population}

U251 cells were transduced with 7TGP and selected by $2 \mu \mathrm{g} / \mathrm{ml}$ puromycin for 1 week. The cells were then cultured in serum-free DMEM medium for $24 \mathrm{~h}$. Then, the cells were treated with serum-free DMEM medium supplemented with different WNT ligands for another $24 \mathrm{~h}$. Cells were washed by PBS and then trypsinized into single cells, analyzed by Flow Cytometer (BD LSRFortessa Cell Analyzer). To enrich Wnt ${ }^{\text {high }}$ or Wnt ${ }^{\text {low }}$ cells in response to WNT3A, cells were treated in serum-free DMEM medium supplemented with $20 \mathrm{ng} / \mathrm{ml}$ WNT3A for $24 \mathrm{~h}$. After $24 \mathrm{~h}$, cells were washed by PBS and sorted by Flow Cytometer (BD FACSAria II Cell sorter) in FITC channel. Wnt ${ }^{\text {high }}$ cells were sorted from the highest $5 \%$ $\mathrm{GFP}^{+}$cells and Wnt ${ }^{\text {low }}$ cells were sorted from the lowest $5 \% \mathrm{GFP}^{+}$cells. After sorting, cells were changed back to normal GBM medium, and allowed to grow for another two passages. Afterward, the sorting was repeated for 4-5 times.

\section{MTT assay}

3000-6000 cells were seeded in $200 \mu \mathrm{l}$ medium in a well of a 96-well plate. Cells were cultured to $60 \%$ confluence in normal medium, then medium was changed to serumfree DMEM medium $24 \mathrm{~h}$ before experiment began. After that, cells were cultured in serum-free DMEM medium containing different Wnt ligands (all at $20 \mathrm{ng} /$ $\mathrm{ml}$ ). MTT assay were performed according to the protocol recommended by the Vendor.

\section{Holoclone assay}

Cells were trypsinized into single cells and seeded at 100-500 cells per well in 6-well plate. Cells were cultured for 14 days, and medium was changed every 3 days. At the end of the experiment, cells were fixed by methanol at room temperature and stained with $0.5 \%$ Crystal Violet for $5 \mathrm{~min}$ at room temperature. Colonies were classified into holoclones, meraclones and paraclones according to their morphology.

\section{Soft agar assay}

The lower layer of agar was mixed by equal volume of $2 \times$ DMEM medium containing $1.2 \%$ agar solution. This mixture was added into each well of a 6-well plate immediately. The upper layer of agar was carefully mixed by adding equal volume of $2 \times$ DMEM medium and $0.6 \%$ agar solution, as well as $3000-8000$ cells. $1.5 \mathrm{ml}$ normal glioma medium was then added in each well, and medium was changed every 3 days for 10-14 days. At the end of experiment, cells were fixed by methanol at room temperature and stained with $0.5 \%$ Crystal Violet for $5 \mathrm{~min}$. The plate was scanned, from which colony numbers were counted.

\section{Sphere formation assay}

2000-3000 single cells were cultured in GSC medium in one well of an ultra-low attachment 6-well plate. Medium was changed every 3 days by centrifuging spheres at $1000 \mathrm{rpm}$ for $2 \mathrm{~min}$. Spheres were counted after 10-14 day culture. To determine the self-renewal ability, spheres were trypsinized and replated at the concentration of 1000 cells $/ \mathrm{ml}$ for another 10 days. After that, spheres were fixed by $70 \%$ ethanol, both sphere numbers and diameters were calculated.

\section{Transwell cell migration assay}

Cells were allowed to grow to $70-80 \%$ confluence, then treated with serum-free DMEM medium for $24 \mathrm{~h}$. Then, cells were trypsinized into single cells and counted. $2 \times 10^{4}$ cells were seeded in the upper chamber whereas $0.5 \mathrm{ml}$ DMEM medium with $10 \% \mathrm{FBS}$, or Wnt ligands was added in the lower chamber. Cells were allowed to migrate into the lower well for $24 \mathrm{~h}$. After $24 \mathrm{~h}$, upper wells were fixed in methanol for 5-10 min at room temperature, and then stained with $0.5 \%$ Crystal Violet for $5 \mathrm{~min}$. In each group, triplicates were used.

\section{$R A$ induced neural differentiation}

3000 GSCs were allowed to form neurospheres for 10 days (GSC medium was changed every 3 days), then the spheres were seeded in DMEM serum-free medium 
containing $10 \mu \mathrm{m} \mathrm{RA}$ in the presence or absence of Wnt ligands for 24-48 h. In addition, one group without RA was used as control representing undifferentiated state.

\section{FACS analysis of CD133 expression}

In brief, cells were digested with either trypsin (for adhesive cells) or Cell Dissociation Buffer (enzyme-free, Thermo Fisher, for suspended cells). Then, 1:20 CD133APC conjugated antibody was dissolved in binding buffer. Up to $1 \times 10^{6}$ cells were suspended with antibody in $200 \mu$ l binding buffer at $4{ }^{\circ} \mathrm{C}$ for $15 \mathrm{~min}$ in dark. After antibody incubation, cells were washed twice by PBS at $1000 \mathrm{rpm}$ for $3 \mathrm{~min}$. Then the cells were analyzed by Flow Cytometer in APC channel. Mouse IgG-APC was used as negative control.

\section{Quantitative real-time PCR}

TRIzol Reagent (Thermo Fisher Scientific) was used to extract total RNA. High-Capacity cDNA Reverse Transcription Kit (Invitrogen) was used to synthesize cDNA from $2 \mu \mathrm{g}$ RNAs per reaction $(20 \mu \mathrm{l})$, according to manufacturer's instruction. For real-time assay, miScript SYBR Green PCR Kit (Qiagen, Germantown, MD) was strictly applied according to manufacturer's instruction. ABI QuantStudio 7 (QS7) Flex Real Time PCR System (384well) was used for amplification. Real-time data was analyzed by ABI QuantStudio 7 (QS7) Flex Real Time PCR System Station. Primer used were summarized in Additional file 2: Table S1.

\section{Western blot}

Protein samples were extracted by RIPA buffer, and $60 \mu \mathrm{g}$ was separated on a $10 \%$ SDS-PAGE gel which subsequently transferred onto a PVDF membrane (Sigma-Aldrich). After blocking with $4 \%$ milk at room temperature for $1 \mathrm{~h}$, the membrane was incubated with primary antibody overnight at $4{ }^{\circ} \mathrm{C}$ on a horizontal rotor. Membrane was washed three times with TBST and incubated with secondary antibodies at room temperature for $1 \mathrm{~h}$. Later, the blot was subjected to chemiluminescent detection with ECL Detection Reagent (Amersham GE Care), and was scanned for analysis. Antibodies used were summarized in Additional file 3: Table S2.

\section{Immunohistochemistry staining}

Tumor tissues were fixed in $4 \%$ paraformaldehyde PBS solution at $4{ }^{\circ} \mathrm{C}$ for $24 \mathrm{~h}$ and then embedded in paraffin. Tissues were cut into $6 \mu \mathrm{m}$ sections and de-paraffined three times in xylene and rehydrated in gradient alcohols. Endogenous peroxidase activity was quenched with $3 \%$ $\mathrm{H}_{2} \mathrm{O}_{2}$ in PBS for $30 \mathrm{~min}$ at room temperature, and sections were washed in PBS 5 min for three times. The sections were heated by microwave at $98{ }^{\circ} \mathrm{C}$ for $20 \mathrm{~min}$ in
$10 \mathrm{mM}$ citrate buffer ( $\mathrm{pH}$ 6.0) for antigen retrieval (PT Module, Thermo). Sections were blocked with 5\% horse serum for $30 \mathrm{~min}$ at room temperature and incubated with primary antibodies at $4{ }^{\circ} \mathrm{C}$ overnight. Later, radish peroxidase-conjugated secondary antibodies (rabbit or mouse, Santa Cruz) were incubated at room temperature for $1 \mathrm{~h}$. Sections were developed with diaminobenzidine and counterstained with hematoxylin using standard protocol.

\section{Xenograft formation}

Nude mice were provided by the Laboratory Animal Service Center of the Chinese University of Hong Kong. They were maintained in an air-conditioned room with controlled temperature of $24 \pm 2{ }^{\circ} \mathrm{C}$ and humidity of $55 \pm 15 \%$, in a $12 \mathrm{~h}$ light/darkness cycle regulation and were fed laboratory chow and water ad libitum. All animal experiments were conducted in accordance with the University Laboratory Animals Service Center's guidelines on animal experimentation with approval from the Animal Ethnics Committee of the University. Female nude mice between 4 and 6 week-old were used in the experiments. $100 \mu \mathrm{l}$ PBS containing different cell numbers (from $1 \times 10^{4}$ to $1 \times 10^{6}$ ) was injected at the flanks of the mice. After inoculation, the condition of nude mice was checked on a daily basis, and tumor volume was recorded using formula $\mathrm{V}=\left(\mathrm{a} \times \mathrm{b}^{2}\right) / 2$, where a represents the longest side of tumor and $b$ represents the width of tumor. Mice with tumor volume larger than $1.5 \mathrm{~cm}^{3}$ or sign of suffer were sacrificed immediately. At the end of the experiment, mice were sacrificed by $\mathrm{CO}_{2}$, and tumors were carefully dissected. Tumor samples were collected for RNA extraction, protein extraction or frozen section.

\section{Statistical analysis}

For in vivo tumor growth curve, one-way ANOVA was used to compare changes in different groups along time. Two-tailed Student's $t$ test was used to compare differences between experimental groups. In each group, data were triplicated or indicated elsewhere, and each bar represents mean $\pm \mathrm{SD}$. Stars on each bar represents statistical significance compared to control group, and additional comparisons were indicated with line segments ("p $<0.05,{ }^{* * *} \mathrm{p}<0.01,{ }^{* * * *} \mathrm{p}<0.001,{ }^{* * * * * *} \mathrm{p}<0.0001$, ns for no significant difference).

\section{Results}

Rspo 2 potentiates Wnt/ $\beta$-catenin signaling in GBM cell lines

We first determined the expression of $\mathrm{RSPO} 2, \mathrm{RSPO} 3$ and their receptors LGR4-6 in GBM cell lines U251 and $\mathrm{U} 87$ by real-time PCR. The results showed that while the expression level of $\mathrm{RSPO} 2$ was comparable 
between U87 and U251, RSPO3 was more highly expressed in U251. In addition, the expression levels of LGR4, LGR5 and LGR6 were higher in U251 than that in U87 (Fig. 1a). Next, U251 or U87 cells were exposed to recombinant Wnt3A, Rspo2, Rspo3 or their combination for $24 \mathrm{~h}$, and examined for the expression levels of $\beta$-catenin target genes. Our results showed that while Wnt3A, Rspo2 or Rspo3 alone had no or mild effect on the expression levels of Axin2, LEF1, cMET, $c-M y c$ or MMP7 in U251 cells, the combination of Wnt3A and Rspo2 dramatically up-regulated the expression levels of the $\beta$-catenin target genes, indicating Rspo2 potentiates the Wnt3A-mediated activation of $\beta$-catenin signaling (Fig. 1b). This induction effect, however, is transient, since neither Rspo2 or Wnt3A alone, nor the combination treatment had any effect at $48 \mathrm{~h}$ (data not shown). It should be noted that Rspo3 did not have potentiation effect on Wnt/ $\beta$-catenin signaling in U251 cells, possibly due to the high intrinsic basal level. In U87 cells, the combination of Rspo2 and Wnt3A significantly enhanced the effect of Wnt3A or Rspo2 alone on LEF1 and MMP7 (Additional file 1: Fig S1A), albeit the extent of this effect was much smaller than that in U251 cells.

Additionally, the combination of Wnt3A and Rspo2 dramatically increased the expression of RSPO2, RSPO3 and LGRs in U251, with the most profound effects observed in RSPO2 and LGR5 (Fig. 1c). Interestingly, while Rspo3 alone mildly increased the expression of RSPO2 and LGRs, the combination of Wnt3A and Rspo3 diminished this effect (Fig. 1c), which is consistent with the effect of Rspo3/Wnt3A on $\beta$-catenin targets (Fig. 1b). In U87 cells, while Rspo2 did not have any effect on the expression levels of RSPO2 and LGRs, the combination of Wnt3A and Rspo2 significantly increased the expression levels of RSPO2 and LGRs, albeit at much lower level compared to that in U251 cells (Additional file 1: Fig S1B). To further validate the potentiation role of Rspos in canonical Wnt signaling in GBM cells, we transduced U251 cells with 7TGP lentiviral vector (Additional file 1: Fig S1C) and demonstrated that the combination of Wnt3A and Rspo2 dramatically enhanced the activation of eGFP compared to Wnt3A or Rspo2 alone (Fig. 1d). Consistent with eGFP expression, the expression level of active $\beta$-CATENIN was significantly increased in the combination treatment compared to Wnt3A or Rspo2 alone. Moreover, the expression levels of CCND1 and AXIN2 also showed the similar enhancement (Fig. 1d). Altogether, these data clearly demonstrate that Rspo2 enhances Wnt3A-activated $\beta$-catenin signaling in GBM cells.

\section{Combination of Rspo2 and Wnt3A upregulates stem cell genes in GBM cells}

Having established that Rspo 2 potentiates $W n t / \beta$-catenin signaling in GBM cells, we tried to determine the functional role of Rspo2 in GBM cells. Surprisingly, neither Wnt3A nor Rspos had any effect on cell growth in U251 or U87 cells. The combination of Wnt3A and Rspos did not have any effects on cell growth either (Fig. 2a). We further determined the migratory ability, which is indicative of malignant characteristics of GBM. Interestingly, while Wnt3A or Rspos alone promoted cell migration, the combination treatment significantly aggravated this effect. Consistent with the previous results, the effect of Rspo2 was stronger than Rspo3 (Fig. 2b).

In GBM and other tumors, the expression and regulation of embryonic stem cell markers appear to be critical for the maintenance of tumor-initiating cells and cancer malignancy [39]. Thus, we went further to determine the effect of Rspos on the expression levels of pluripotent markers in GBM cell lines. Our real time PCR showed that while Wnt3A alone did not affect the expression of pluripotent stem cell markers, the combination of Wnt3A and Rspo2 dramatically increased the expression of OCT4, NANOG, SOX2 and KLF4 in U251 cells. In contrast, neither Rspo3 nor Wnt3A/Rspo3 groups had any effects on pluripotent stem cell markers (Fig. 2c). In U87 cells, both Wnt3A and Rspo2 dramatically increased the mRNA expression of CD133 and SOX2. However, the effect of Wnt3A or Rspo2 could not be potentiated by the combination therapy. On the other hand, while Wnt3A and Rspo2 alone did not affect NANOG expression, the combination of Wnt3A and Rspo2 dramatically increased the expression of NANOG (Additional file 1: Fig S2A). Given that GBM are heterogeneous and hierarchically organized with GSCs at their apex, the upregulation of stem cell markers could be due to the enrichment of GSCs or the enhancement of GSC stemness. Our flow cytometry analysis demonstrated that neither Wnt3A or Rspo2 alone nor the combination treatment had any effect on the enrichment of $\mathrm{CD} 133^{+}$positive cells in U251 (Additional file 1: Fig S2B), suggesting that Rspo2/ Wnt3A enhances stem cell traits in GSCs rather than enlarges the GSC pool within GBM cells.

\section{Rspo/Lgr and Wnt/ $\beta$-catenin signaling are over-activated in GSC-like cells}

Next, we enriched for GSC-like cells from U251 and U87 cell lines using the well-established GSC culture condition [40, 41]. After 2 months of enrichment, we detected the CD133 positivity in U251 and U87 GSC-like cells and found that more than $63 \%$ cells were $\mathrm{CD}_{133^{+}}$in $\mathrm{U} 251$ whereas $35.3 \%$ cells were $\mathrm{CD}_{133^{+}}$in U87 (Additional 

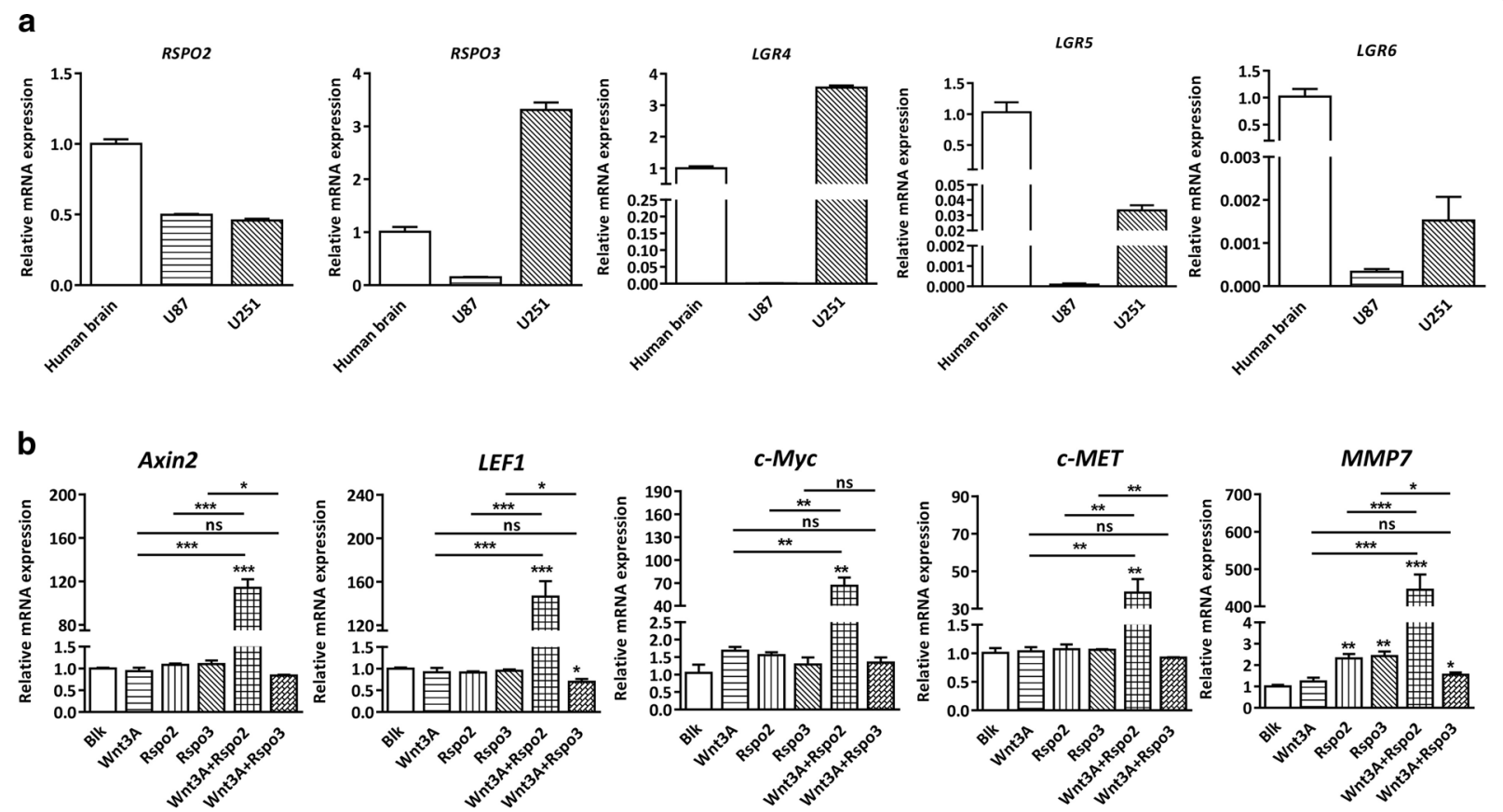

C
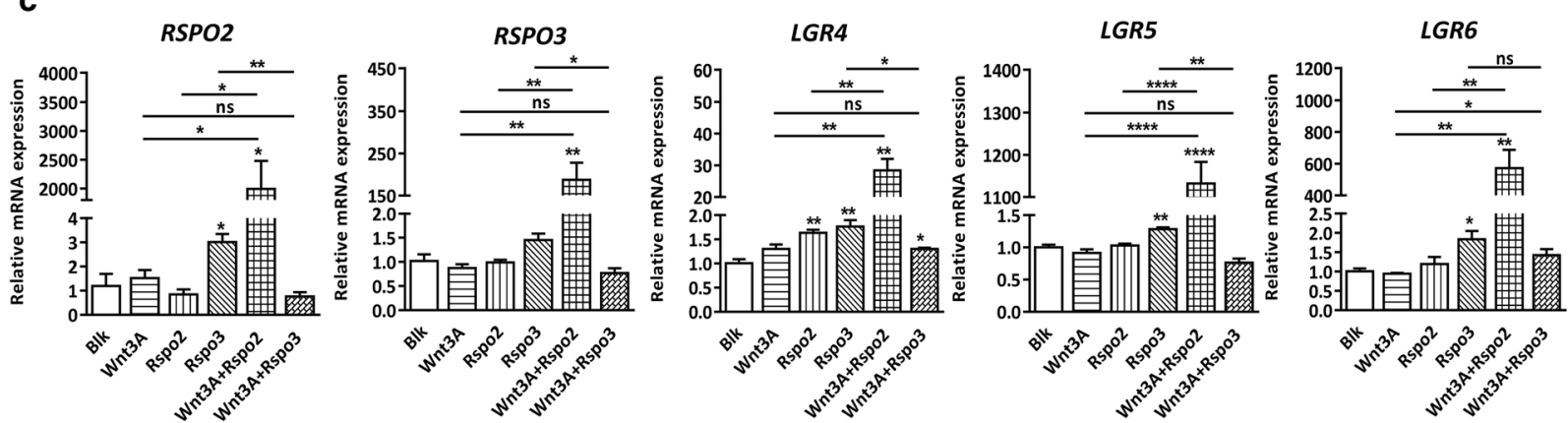

d

\section{d}

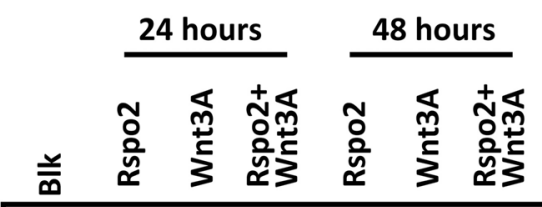

active $\beta$-CATENIN

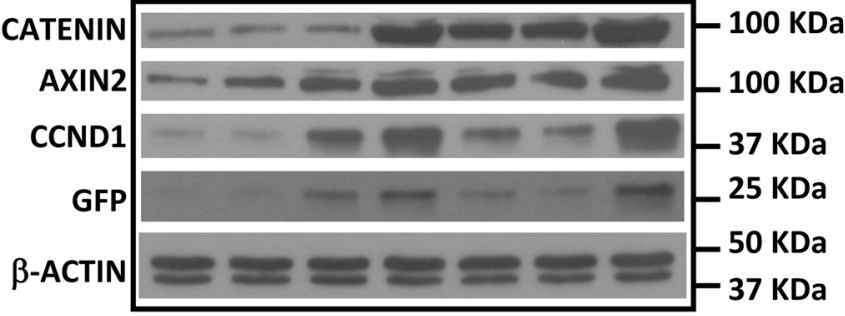

Fig. 1 Expression pattern and potentiation effect of RSPOs in U251 cells. a mRNA expression levels of RSPO2/3 and their receptors LGR4-6 were determined in U87 and U251 cells. U251 cells were pre-treated in serum-free medium for $24 \mathrm{~h}$, then cultured in serum-free medium containing different Wnt ligands (20 ng/ml Wnt3A, Rspo2, Rspo3) for another $24 \mathrm{~h}$. Rspo2 shows prominent potentiation effect on Wnt3A-induced canonical $\beta$-catenin downstream targets (b), and RSPO-LGR genes (c). Blk indicates U251 cells cultured in serum-free medium containing $0.1 \%$ DMSO. d 7TGP-transduced U251 cells were pre-treated in serum-free medium for $24 \mathrm{~h}$, then cultured in serum-free medium containing different Wnt stimuli for another 24-48 h. Representative Western Blot showing the protein expression levels of canonical $\beta$-catenin downstream targets in 7TGP-transduced U251 cells with Wnt ligand treatment 
file 1: Fig S3A). The GSC properties were confirmed by both sphere formation assay and holoclone assay, results of which showed enhanced sphere forming capability and increased number of holoclones in the GSC-like cells (Additional file 1: Fig S3B, C). To determine whether these GSC-like cells are endowed with differential Wnt/ $\beta$-catenin activity compared to their parental cells, we examined the mRNA expression levels of $\beta$-catenin target genes and RSPOs/LGRs in U251 and U87 GSCs. Our results showed that comparing to U251 parental cells, the mRNA expression levels of $\beta$-catenin targets, such as Axin2, LEF1, $c-M E T$ and $c-M y c$ were dramatically increased in the GSC-like cells (Fig. 3a). In addition, the expression levels of Nestin and SOX2 were also markedly up-regulated in the GSC population, confirming that GSC-like cells share NSC-associated traits. Besides, the expression levels of RSPO2, RSPO3 and LGRs were globally upregulated in U251 GSCs compared to parental cells, indicating that the Rspo/Lgr axis is over-activated in GSC-like cells (Fig. 3b). Consistently, in U87 GSCs, the mRNA expression levels of $\beta$-catenin targets and NSC marker, as well as RSPO2, RSPO3 and LGR4 were markedly increased compared to parental cells (Additional file 1: Fig S3D, E). Next, we determined the protein expression levels of active $\beta$-CATENIN, $\beta$-catenin target genes and LGRs in U251 and U87 GSCs in relative to their parental cells. In line with the mRNA data, the expression levels of active $\beta$-CATENIN and its targets were dramatically increased in the GSC-like cells. In addition, the expression of LGR4 and LGR5 was also significantly increased in the GSC-like cells compared to their parental cells (Fig. 3c).

\section{Rspo2 and Wnt3A maintains the stemness of GSC-like cells}

Next, we asked what is the role of Rspo2 in GSC-like cells? To answer this question, we evaluated the effect of Wnt3A and/or Rspo2 on stem cell marker induction. As shown in Fig. 4a, while Wnt3A or Rspo2 alone significantly increased the expression of GSC markers such as OCT4 and Nestin, the combination of Wnt3A and Rspo2 aggravated the induction effect. For NANOG, neither Wnt3A nor Rspo2 alone increased the expression, however, combined treatment with Wnt3A and Rspo2 dramatically induced the expression. To further demonstrate the role of Wnt3A and Rspo2 in maintaining the stem cell traits of GSCs, we went further to examine the effects of Wnt3A and Rspo2 on preventing neural differentiation of GSCs. In this set of experiments, GSC-like cells were cultured as neurospheres, and then seeded in serumdeprived medium containing $10 \mu \mathrm{m}$ Retinoid Acid (RA) in the presence or absence of Wnt3A and/or Rspo2. RA treatment induced GSC differentiation robustly as demonstrated by dramatic downregulation of stem cell markers including CD133, Nestin and SOX2 and induction of differentiation markers such as TUJ1 and GFAP. Intriguingly, while Wnt3A or Rspo2 alone exhibited mild effect, the combination of Wnt3A and Rspo2 completely rescued the downregulation of stem cell markers and upregulation of differentiation markers (Fig. 4b). The experiments were repeated in U87 GSCs, which revealed the similar results (Additional file 1: Fig S4A). It has been well established that GSCs can be maintained in EGF- and FGF2-enriched culture condition to sustain their stemness. To further consolidate our finding that Wnt3A and Rspo2 is critical for maintaining the stemness of GSCs, we cultured the U251 GSC-like cells without EGF and FGF2 in the absence or presence of Wnt3A and Rspo2. As expected, the deprivation of EGF and FGF2 significantly decreased the diameters and numbers of neurosphere, however, this effect could be significantly reversed by the addition of Wnt3A and Rspo2 (Fig. 4c, Additional file 1: Fig S4B). In addition, grow factor deficiency-induced suppression of stem cell markers could be completely abolished by the co-treatment with Wnt3A and Rspo2 (Fig. 4d). On the other hand, it should be noted that withdrawal of EGF and FGF2 led to a marked suppression of $\beta$-catenin signaling (Additional file 1: Fig S4C), which was reversed by the combination of Wnt3A and Rspo2. Altogether, these results clearly demonstrate the essential role of Rspo 2 and $\mathrm{Wnt} / \beta$-catenin signaling in the prevention of GSC differentiation and maintenance of their stemness.

\footnotetext{
(See figure on next page.)

Fig. 2 Combination of Rspo2 and Wnt3A does not affect cell growth, but elevates the expression levels of stem cell genes in U251 cells. a U87 and U251 cells were pre-treated in serum-free medium for $24 \mathrm{~h}$, then cultured in serum-free medium containing different Wnt ligands for another 4 days, and cell growth was determined by MTT assay. Results show neither WNT ligand alone nor in combination has any effect on cell growth. $\mathbf{b}$ Transwell migration assay was performed in U251 cells. U251 cells were pre-treated in serum-free medium for $24 \mathrm{~h}$, then $2 \times 10^{4}$ cells were cultured in serum-free medium in chambers, while wells containing different Wnt stimuli were used as chemotaxis source for another $24 \mathrm{~h}$. scale bar represents $200 \mu \mathrm{m}$. Quantification analysis of data is expressed as the Mean \pm SD from triplicates of two independent experiments. c The mRNA expression levels of stem cell marker OCT4, SOX2, NANOG and KLF4 were determined by real time PCR, results show that the combination of Rspo2 and Wnt3A significantly upregulates stem cell markers
} 


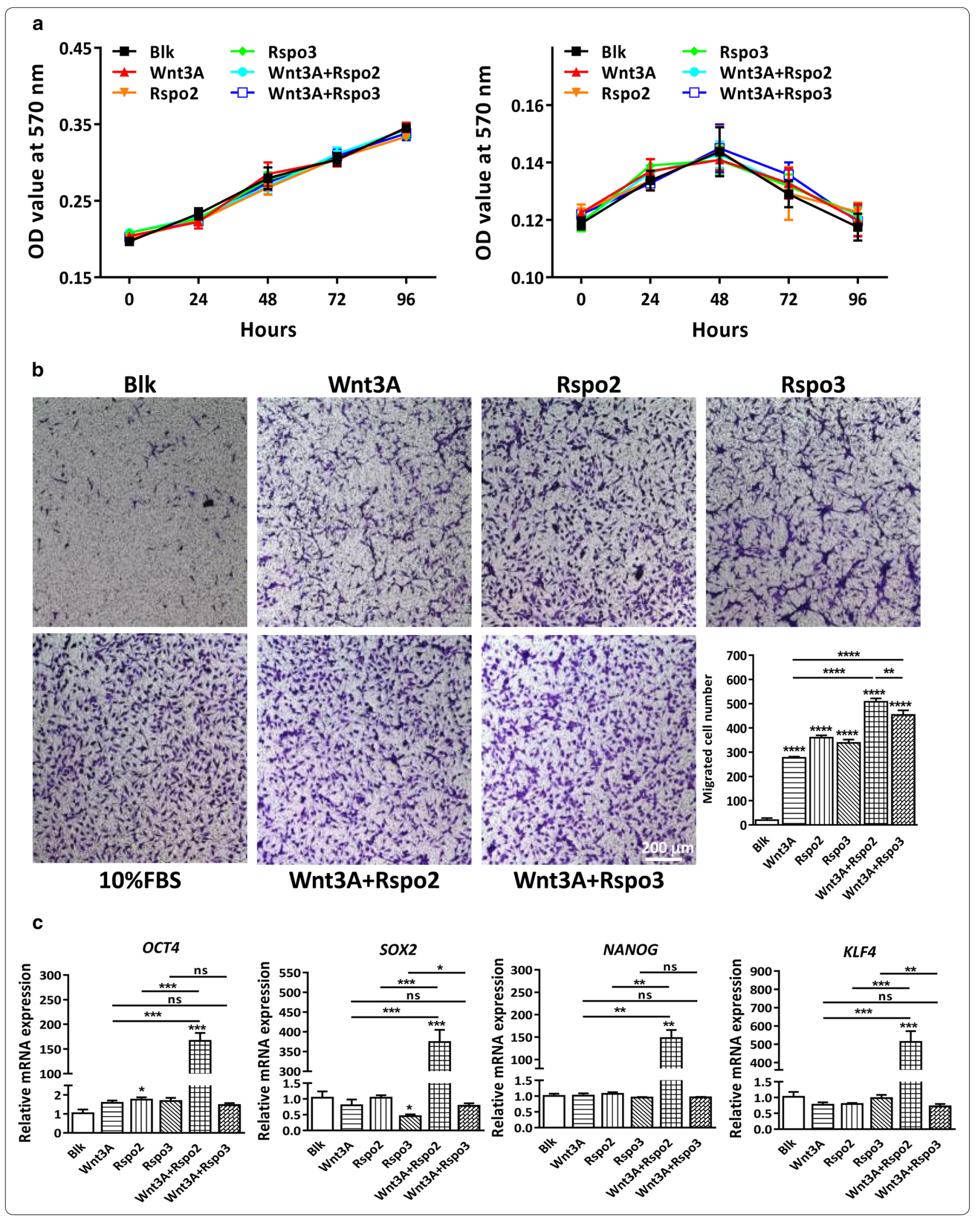




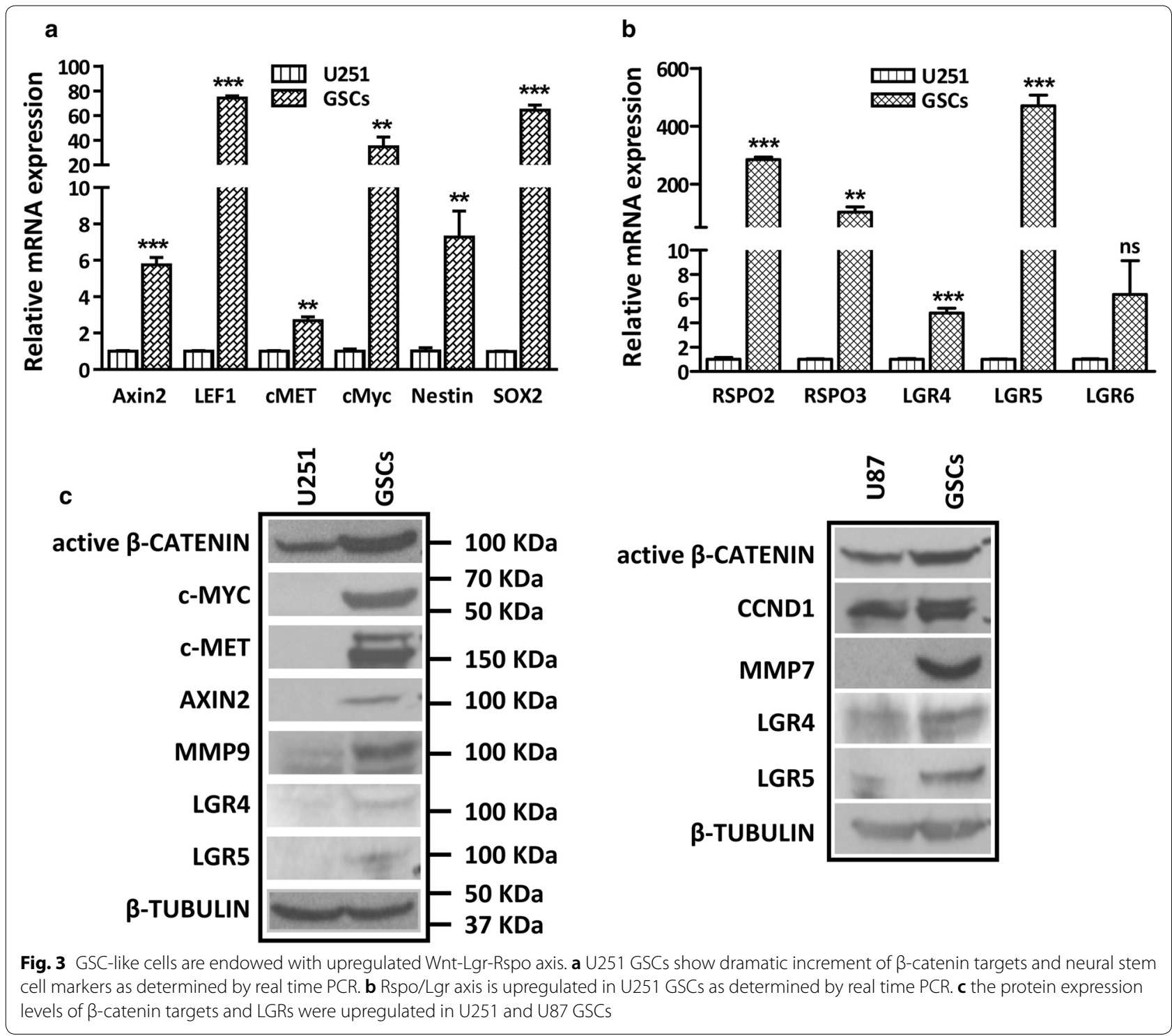

Wnt/GFPhigh and Wnt/GFPlow cell populations display distinct biologic properties

Given that activation of $\mathrm{Wnt} / \beta$-catenin signaling is instrumental for GSC traits, we reasoned that the different responsiveness of GBM cells to Wnt ligands could contribute to GSC property. To prove this hypothesis, U251 7TGP stable cells were cultured in serum-free medium containing $20 \mathrm{ng} / \mathrm{ml}$ Wnt3A for $24 \mathrm{~h}$. Subsequently, Wnt ${ }^{\text {high }}$ cells were sorted from the highest $5 \% \mathrm{eGFP}^{+}$cells and Wnt ${ }^{\text {low }}$ cells were sorted from the

\footnotetext{
(See figure on next page.)

Fig. 4 Wnt3A and Rspo2 maintains stemness in GSCs. a Rspo2 and Wnt3A upregulates stem cell marker in U251 GSCs as demonstrated by real time PCR. BIk indicates U251 GSCs cultured in GSC media with 0.1\% DMSO. b All-trans retinoic acid (10 $\mu \mathrm{M}$ RA) was used to induce differentiation in U251 GSCs for $24 \mathrm{~h}$ with or without Wnt ligands $(20 \mathrm{ng} / \mathrm{ml})$. Real-time PCR was used to determine the effect on differentiation. Results show that Rspo2/ Wnt3A treatment rescues RA-induced GSC differentiation. Blk indicates U251 GSCs cultured in DMEM with 0.1\% DMSO. c GSCs were cultured in GSC media, or GSC media without EGF and FGF, or GSC media without EGF and FGF but with Wnt3A and Rspo2 for 7 days. Results show that lack of EGF/ FGF causes reduced sphere formation, which can be compensated by adding Rspo2/Wnt3A. d Real-time PCR shows that Rspo2/Wnt3A treatment abolishes the downregulation of stem cell markers and upregulation of differentiation markers caused by growth factor deprivation. Blk in C and D indicates U251 GSCs cultured in GSC media with $0.1 \%$ DMSO
} 


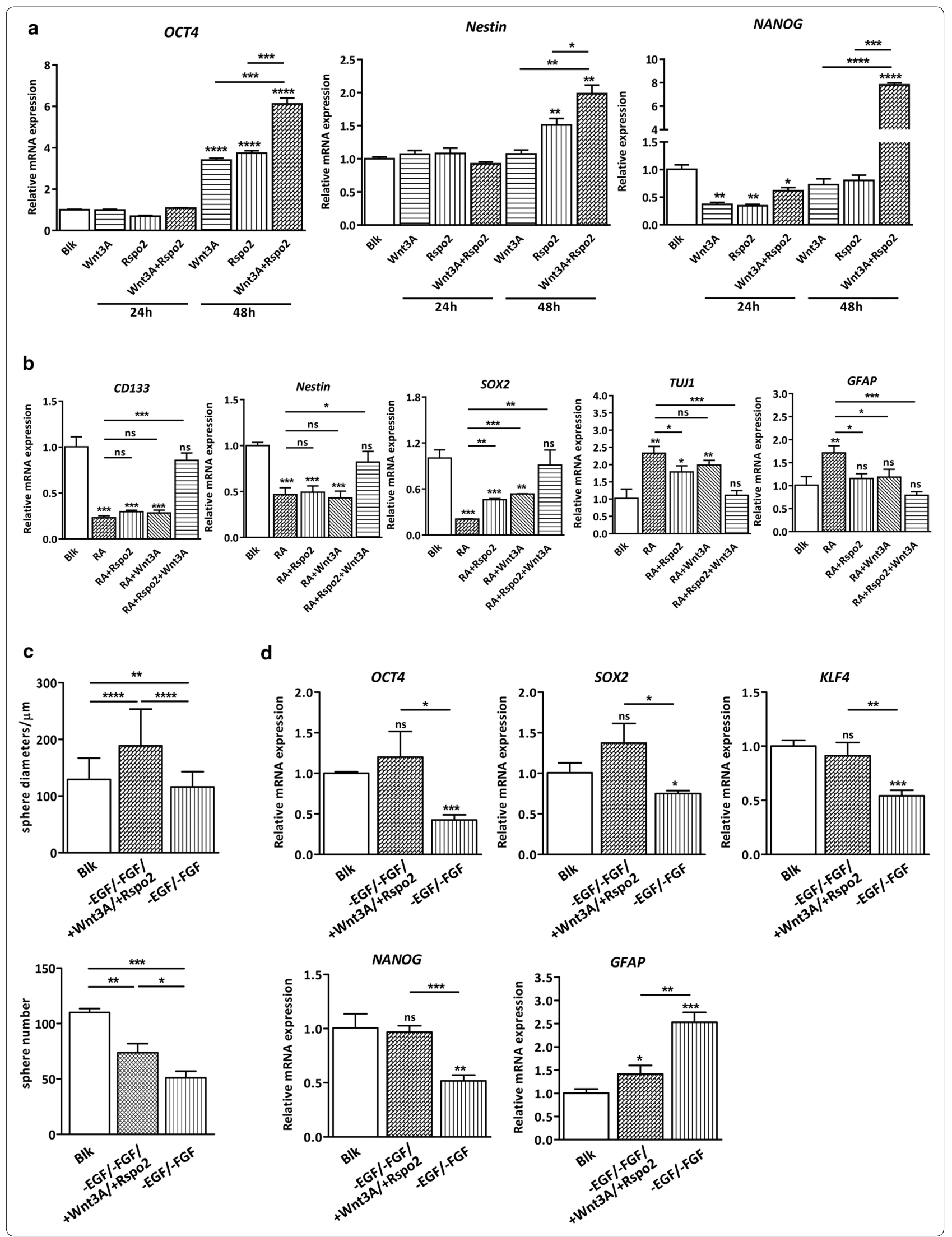


a

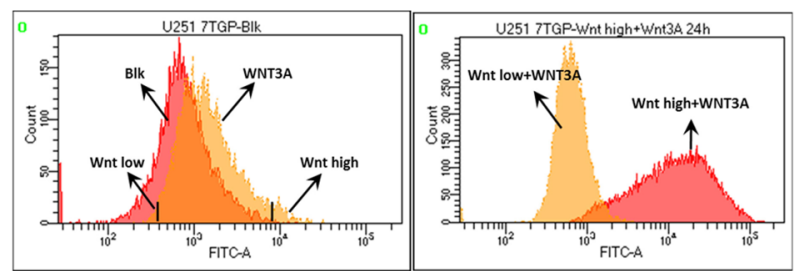

b

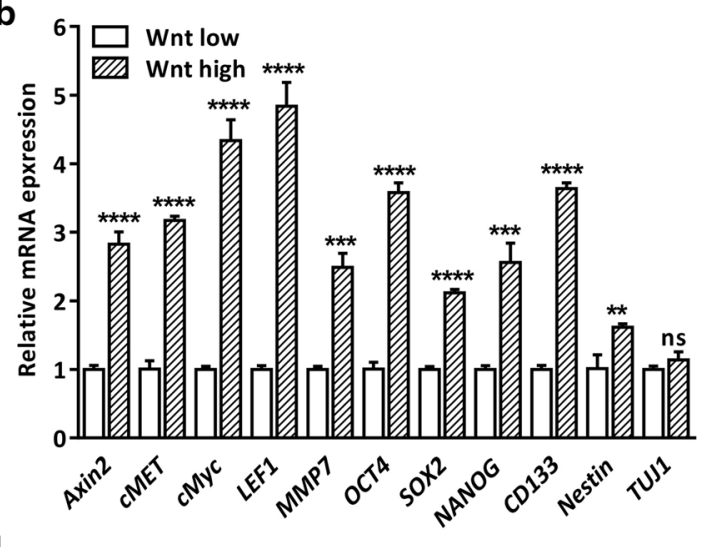

d
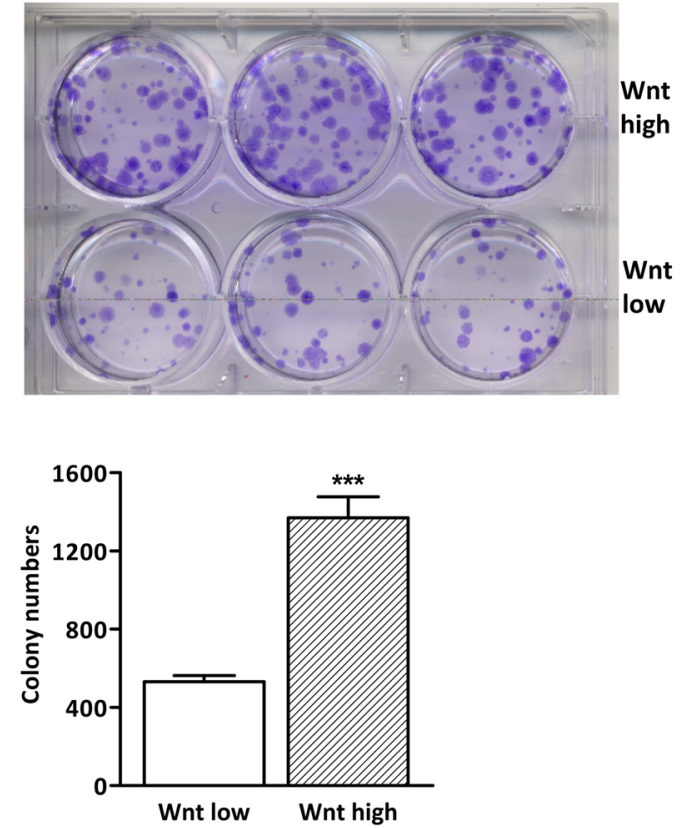

C
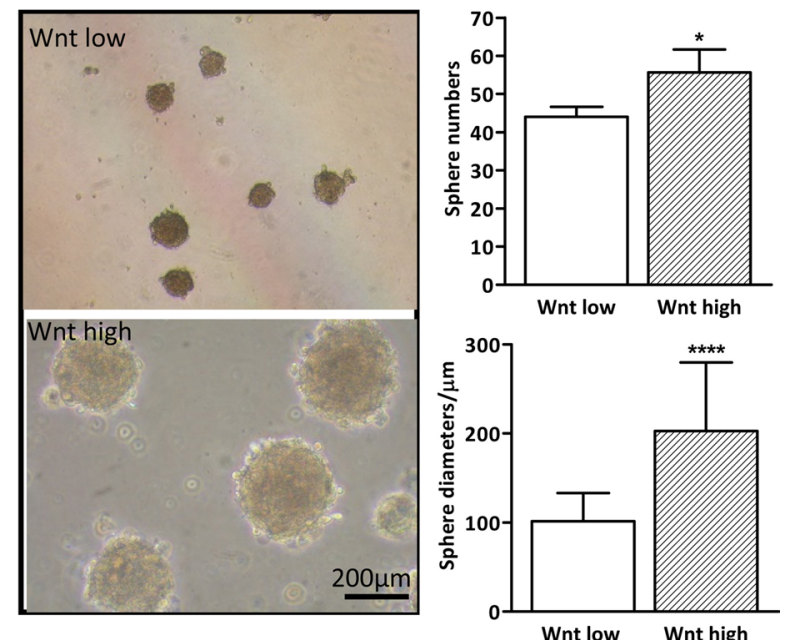

e

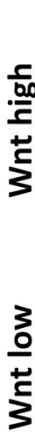

NC
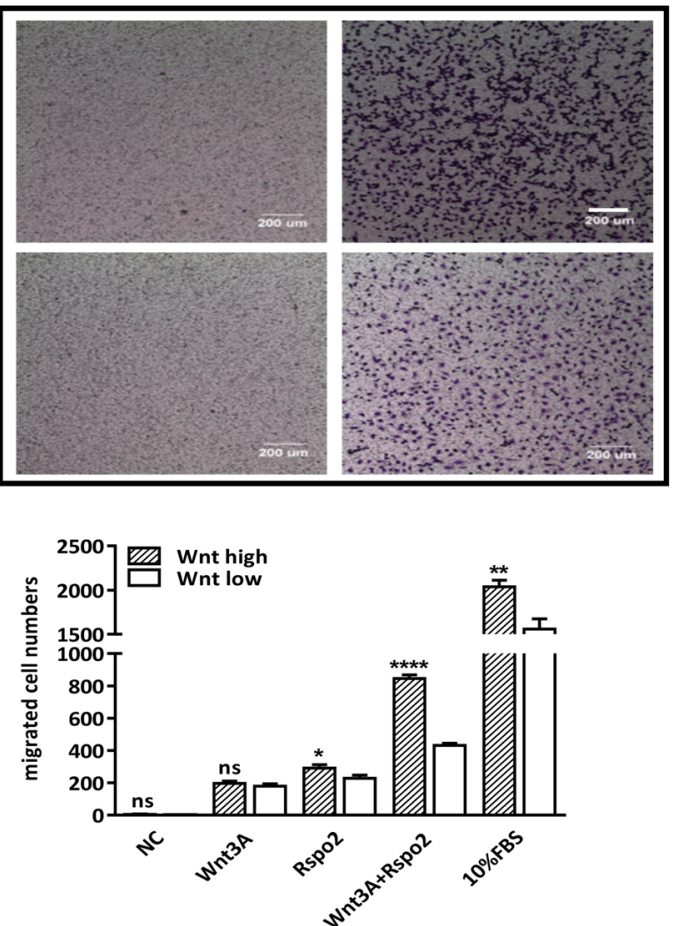

Fig. 5 Wnt ${ }^{\text {high }}$ and Wnt ${ }^{\text {low }}$ sub-populations of U251 cells exhibit different cellular properties in vitro. a lsolation of Wnt ${ }^{\text {tigh }}$ and Wnt ${ }^{\text {low }}$ sub-populations of U251 cells. Left panel, Typical flow cytometry analysis depicting unsorted U251-7TGP cells treated with $20 \mathrm{ng} / \mathrm{ml} \mathrm{Wnt3A}$ (yellow). For subsequent experiment, the $5 \%$ of cells with the highest and lowest GFP levels were sorted. Right panel, shown is the flow cytometric reanalysis of the respective subgroups after several rounds of enrichment. $\mathbf{b}$ Real-time PCR demonstrates that Wnthigh cells show higher expression levels of $\beta$-catenin targets and stem cell markers. c Sphere formation assay shows that Wnt ${ }^{\text {high }}$ cells have an enhanced sphere formation ability. Scale bar is $200 \mu \mathrm{m}$. Quantification analysis of data is expressed as the Mean \pm SD from three independent experiments. $\mathbf{d}$ Soft agar assay shows Wnt ${ }^{\text {high }}$ cells have a higher anchorage-independent cell growth ability. Quantification analysis of data is expressed as the Mean \pm SD from three independent experiments. e Transwell assay shows Wnt ${ }^{\text {high }}$ cells exhibit enhanced cell migratory ability. Quantification analysis of data is expressed as the Mean \pm SD from three independent experiments. N. C. indicates vehicle control of Wnt ${ }^{\text {high }}$ and Wnt ${ }^{\text {low }}$ cells 
lowest $5 \% \mathrm{GFP}^{+}$cells. After several rounds of enrichment, two subgroups of cells with distinctive response to Wnt3A were established (Fig. 5a). We then evaluated the canonical Wnt activity in the two cell subsets and found that the basal expression levels of the $\beta$-catenin targets (Axin2, LEF1, cMyc, c-MET and MMP7) were significantly increased in GFP/Wnt ${ }^{\text {high }}$ compared to GFP/ Wnt ${ }^{\text {low }}$ cells, indicating a higher level activity of $\beta$-catenin signaling in Wnt ${ }^{\text {high }}$ cells (Fig. 5b). Of interest, we also found that Wnt ${ }^{\text {high }}$ cells displayed significantly increased expression levels of stemness genes including OCT4, SOX2, NANOG, CD133 and Nestin, suggesting distinctive stemness state between Wnt ${ }^{\text {high }}$ and Wnt ${ }^{\text {low }}$ cells. In addition, comparative Western blotting in the two subpopulations corroborated high Wnt responsiveness in Wnt ${ }^{\text {high }}$ cells with upregulated expression of GFP, active $\beta$-CATENIN and $\beta$-catenin targets, such as AXIN2, c-MYC, CCND1, c-MET (Additional file 1: Fig S5A). In addition, the protein expression levels of EMT markers, such as TWIST and VIMENTIN, were also dramatically increased in the Wnt ${ }^{\text {high }}$ cells in response to Rspo2 and/ or Wnt3A, suggesting distinctive malignancy states of the two subpopulations.

Having established the divergent Wnt/GFP status in Wnt ${ }^{\text {high }}$ and Wnt ${ }^{\text {low }}$ U251 cells, we went further to examine the biological properties of those two subpopulations. To our surprise, we did not find any significant difference in anchorage-dependent cell growth between Wnt ${ }^{\text {high }}$ and Wnt ${ }^{\text {low }}$ cells, either in untreated status or treated with Wnt3A and/or Rspo 2 (Additional file 1: Fig S5B). We then switched to sphere forming assay and the result showed that more spheres were formed in Wnt ${ }^{\text {high }}$ than that in Wnt ${ }^{\text {low }}$ cells. In addition, Wnt ${ }^{\text {high }}$ cells formed larger spheres than Wnt ${ }^{\text {low }}$ cells (Fig. 5c). Moreover, there were more colony formation with $\mathrm{Wnt}^{\text {high }}$ population than Wnt ${ }^{\text {low }}$ population in soft agar assay (Fig. 5d). We also determined their difference in cell migration and found that Wnt ${ }^{\text {high }}$ cells showed increased migratory capability compared to Wnt ${ }^{\text {low }}$ cells (Fig. 5e), which is in corroboration with the increased expression levels of TWIST and VIMENTIN in Wnt ${ }^{\text {high }}$ cells (Additional file 1: Fig S5A).

\section{Wnt/GFPhigh population possesses increased tumorigenic potential in vivo}

Next, we sought to determine whether difference in WNT responsiveness in GBM cells leads to altered tumorigenic capability in vivo. To do this, Wnt ${ }^{\text {high }}$ or Wnt ${ }^{\text {low }}$ subpopulations were subcutaneously implanted into 6 week old-male nude mice with two concentrations (Additional file 1: Fig S5C). The result showed that all mice formed tumors with $1 \times 10^{5} \mathrm{Wnt}^{\text {high }}$ or $\mathrm{Wnt}^{\text {low }}$ cells. However, while all mice formed tumors with $1 \times 10^{4}$
Wnt ${ }^{\text {high }}$ cells, only 6 out 9 mice formed tumors with $1 \times 10^{4} \mathrm{Wnt}^{\text {low }}$ cells (Additional file 1: Fig S5C). In addition, the tumors grew more slowly with $1 \times 10^{4} \mathrm{Wnt}^{\text {low }}$ cells than that with Wnt ${ }^{\text {high }}$ cells (Fig. 6a). These results indicate that Wnt ${ }^{\text {high }}$ cells have increased tumorigenicity than Wnt ${ }^{\text {low }}$ cells in vivo. Subsequent closer examination of tumor samples demonstrated that the expression levels of activate $\beta$-CATENIN, eGFP and $\beta$-catenin target AXIN2, CCND1 were significantly higher in Wnt ${ }^{\text {high }}$ xenografts compared to Wnt ${ }^{\text {low }}$ xenografts, indicating high Wnt activity is maintained in Wnt ${ }^{\text {high }}$ cell-transplanted tumors. Correspondingly, we found a significantly higher expression of RSPO2 and LGR4 in these tumors as well (Fig. 6b-d). In addition, the expression levels of TWIST and VIMENTIN, which were indicative of higher migratory ability and GSC property, were significantly higher in Wnt ${ }^{\text {high }}$ xenografts. In contrast, the expression level of differentiation marker TUJ1 was significantly lower in Wnt ${ }^{\text {high }}$ xenografts compared to Wnt ${ }^{\text {low }}$ xenografts (Fig. 6b, c). Together, these data suggest a more malignant signature of Wnt ${ }^{\text {high }}$ xenografts.

\section{Discussion}

Taken together, our study has unveiled a previously undefined role of Rspos in potentiating canonical Wnt signaling in GBM, which promotes cancer stemness trait.

In the current study, we have found that Rspo2 maintains GSC traits via potentiating Wnt. Wnt3A/Rspo2 promotes the self-renewal capacity of GSCs and prevents them from RA-or growth factor deprivation-induced differentiation (Fig. 4b-d, Additional file 1: Fig S4). These results clearly indicate that the stem-like characteristics of GSCs can be effectively maintained by the synergistic effect of Rspo2 and Wnt3A, while Rspo2 or Wnt3A alone is not sufficient. Our finding is consistent with the previous study showing that the Wnt-Lgr5-Rspo axis is crucial for maintaining colonic and pancreatic CSCs for long-term in vitro expansion and self-renewal $[32,34]$. On the other hand, one recent study using lineage tracing clearly revealed that Wnt ligands had qualitatively distinct, non-interchangeable roles in intestinal stem cells (ISC) [42]. i.e. Wnt proteins themselves were unable to induce ISC self-renewal, but instead conferred a basal competency by enabling Rspo ligands to actively drive and specify the extent of stem-cell expansion. This functionally non-equivalent yet cooperative interaction mode between Wnt and Rspo may explain the synergistic effect of Wnt3A and Rspo2 on GSCs. Indeed, fine-tuning of Wnt/ $\beta$-catenin activity by Wnt ligands is essential to optimally maintain neoplastic cells and the level of Wnt activation required for this effect is likely to be tissue and cell type specific [43]. It should be noted that GSCs are endowed with intrinsic high activity of Wnt-Lgr-Rspo 


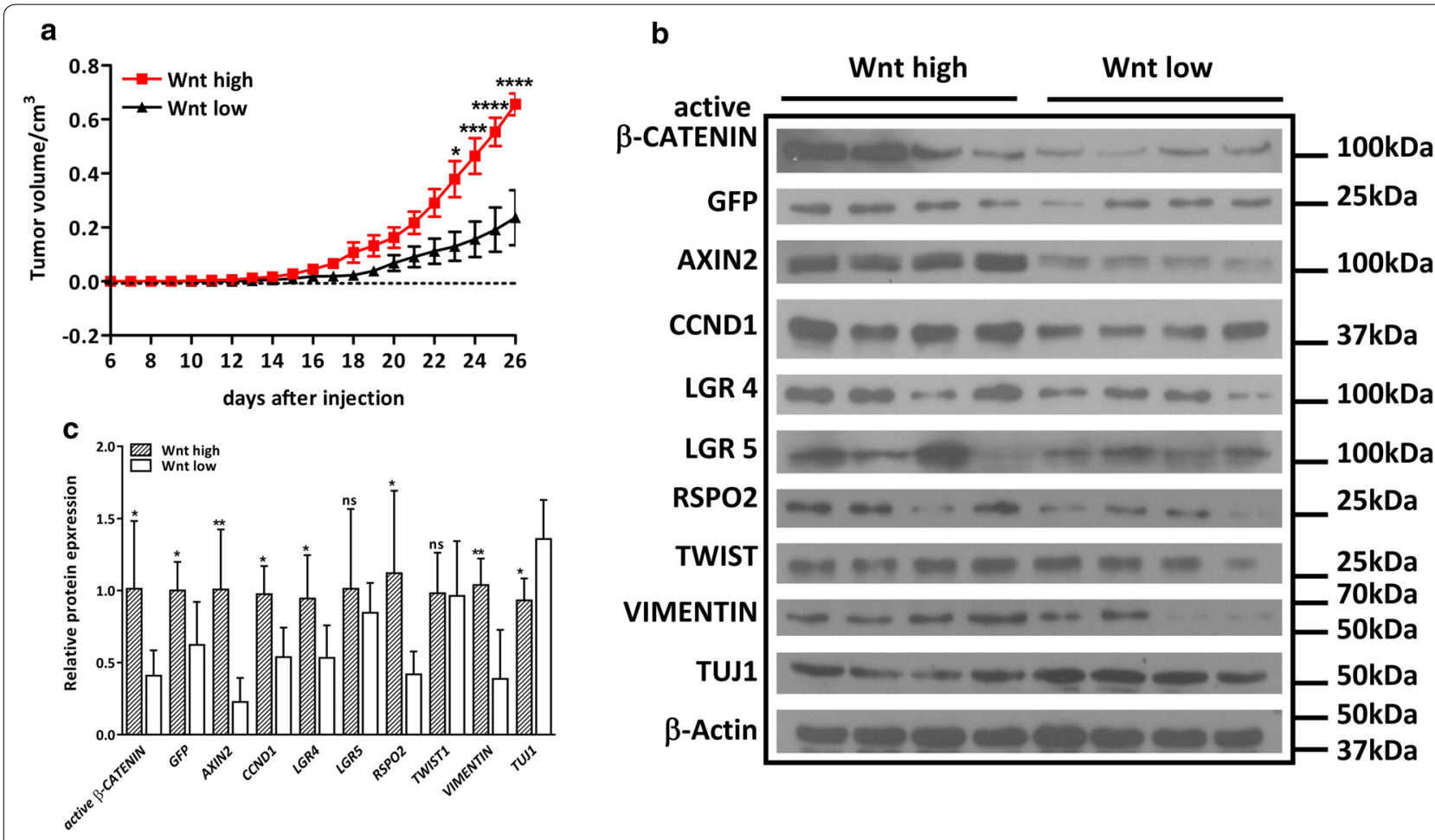

d

Blk

LGR 4

RSPO2

CCND1

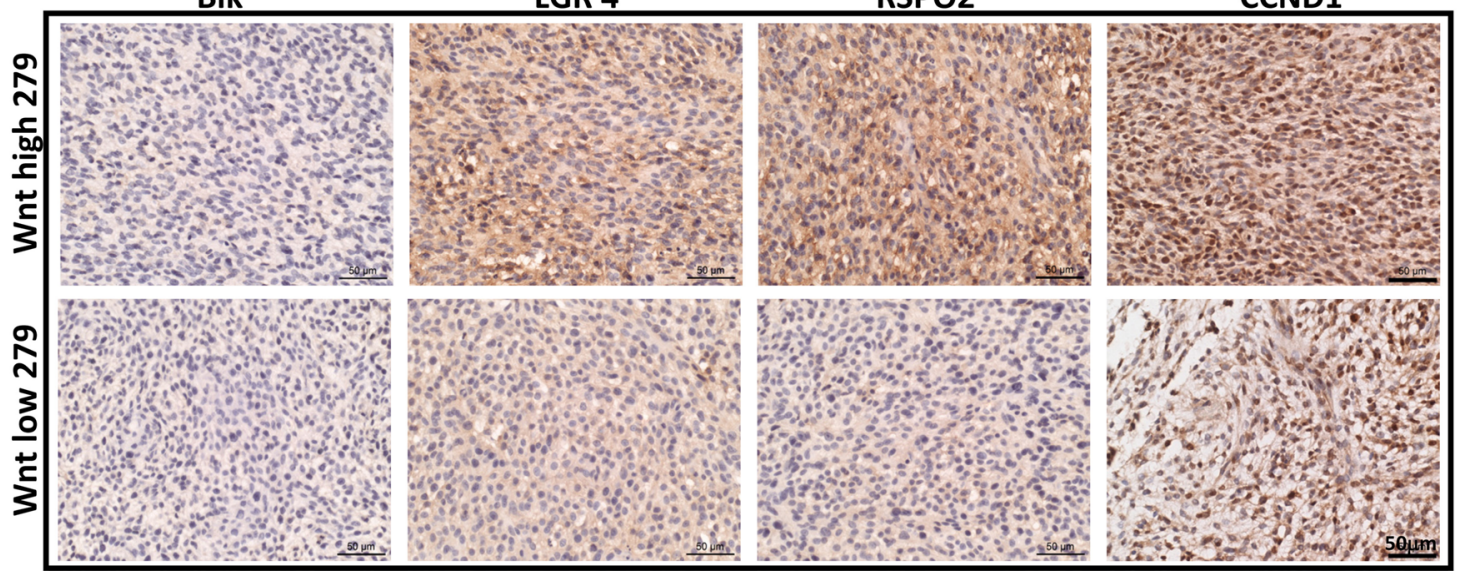

Fig. $6 \mathrm{Wnt}^{\text {high }}$ tumors grow faster and have high RSPO expression. a $1 \times 10^{4} \mathrm{Wnt}^{\text {high }}$ or Wnt ${ }^{\text {low }}$ cells were inoculated at the flanks of nude mice, and tumor growth curve were recorded. The result shows that Wnt ${ }^{\text {high }}$ cell-injected xenografts $(n=9)$ grow faster than Wnt ${ }^{\text {low }}$ cell-injected xenografts $(n=6)$. $\mathbf{b}$ Protein expression of $\beta$-catenin targets, RSPO/LGR, EMT markers and differentiation marker was compared between Wnt ${ }^{\text {high }}(n=4)$ and Wnt ${ }^{\text {low }}(n=4)$ xenografts. $\mathbf{c}$ Statistic analysis of G. d Tumor sections from Wnt ${ }^{\text {high }}$ or Wnt ${ }^{\text {low }}$ xenografts were stained by RSPO2, LGR4 and CCND1, where Wnt ${ }^{\text {high }}$ tumors display stronger staining compared to Wnt ${ }^{\text {low }}$ tumors. Scale bar is $200 \mu \mathrm{m}$

signaling. In particular, the upregulation of Rspo/Lgr axis is prominent, i.e. Rspo2 and Lgr5 are upregulated more than 200- and 500fold respectively in U251 GSCs compared to parental cells (Fig. $3 \mathrm{a}-\mathrm{c}$ ). It is plausible that Rspo2 amplifies Wnt/ $\beta$-catenin signaling and ensures a high and stable Wnt environment surrounding GSCs.
Indeed, previous study has revealed that Rspo/Lgr signaling in ISCs is essential for establishing a Wnt gradient in the ISC niche [44].

Heterogeneous responsiveness to $\mathrm{Wnt} / \beta$-catenin signaling has been observed in various cancers, such as colon cancer and breast cancer $[45,46]$. Reciprocally, 
tumor cells with high $\beta$-catenin activity within a tumor mass appear to undergo EMT and acquire cancer stem cell property [47]. On the other hand, CSC properties can be altered by environmental cues, such as hypoxia and growth factor exposure. Interestingly, in the current study, we have shown that GBM cells exhibit distinctive Wnt ligand responsiveness. Wnt ${ }^{\text {high }}$ responders are more susceptible to extrinsic Wnt stimulation with both Wnt protein and Wnt enhancer. Treatment with Rspo 2 alone or in combination with Wnt3A has mild effects on $\beta$-catenin targets in low-responder cells, whereas high-responders react boldly to Wnt3A, Rspo2, and to their combination (Additional file 1: Fig S6A). In addition, we have clearly demonstrated that Wnt ${ }^{\text {high }}$ responders exhibit a more aggressive malignant phenotype with stem cell traits than Wnt ${ }^{\text {low }}$ responders. First, the Wnt ${ }^{\text {high }}$ population expresses higher stemness genes and EMT markers compared to $\mathrm{Wnt}^{\text {low }}$ population. Second, Wnt ${ }^{\text {high }}$ cells show growth advantage in sphere formation assay and soft agar assay, indicating their enhanced stemness. Third, Wnt ${ }^{\text {high }}$ cells exhibit stronger migratory ability, suggesting a more aggressive phenotype. Finally, Wnt ${ }^{\text {high }}$ cells show enhanced tumorigenicity and grow faster than Wnt ${ }^{\text {low }}$ cells in vivo. In addition, Wnt ${ }^{\text {tigh }}$ xenografts are more malignant than Wnt ${ }^{\text {low }}$ xenografts. Altogether, these results clearly indicate that not only the baseline level of Wnt/ $\mathrm{B}$ catenin, but also the functional response and adaptability to contextual Wnt signals is of great importance for GBM stemness. In fact, the interaction between tumor cells and the surrounding microenvironment can locally affect the intracellular levels of canonical Wnt signaling, which triggers stemness, cell proliferation, EMT and invasive behavior. For instance, Rspo1, which is hormonally regulated in luminal epithelial cells by estrogen and progesterone, acts in concert with Wnt4 to expand mammary stem cells [48]. In addition, Rspo2 was reported to enhance Wnt signaling and stemness in Wnt responsive pancreatic cancer cells [34]. These findings indicate that both intrinsic and extrinsic factors are likely to play critical roles in cancer stemness, local invasion and metastasis by differentially modulating Wnt $/ \beta$-catenin signaling. In our study, RSPO2 protein is found to be overexpressed in Wnt ${ }^{\text {high }}$ GBM xenografts (Fig. 6a-c), which confirms that Rspo2 plays a major role in regulating Wnt signaling in susceptible GBM cells. Nevertheless, it should be noted that RSPO2 and LGR4 are not globally expressed in all tumor cells in the Wnt ${ }^{\text {high }}$ GBM xenografts (Fig. 6d), suggesting heterogeneous property of GBM cells and their distinctive of $\beta$-catenin activity. Future investigation focusing on the link between the heterogeneous expression of RSPO/LGR and cancer stemness in primary GBM tissues is necessary.

During development, Rspo2 is prominently expressed in the apical ectodermal ridge (AER) of the limb bud, and more expression has been detected in the developing lungs, brain, pharynx, teeth, long bones, craniofacial bones, and vertebrae [49]. The major phenotypes associated with the global loss of Rspo 2 during development are limb and craniofacial malformations and hypomorphic lungs, which result in perinatal death $[50,51]$. Rspo3 is highly expressed in the primitive streak during very early development and is also expressed in the developing neural tube, brain, limb bud, heart, kidney, and small intestine. However, embryonic death around E10 limits the investigation of further effects of Rspo3 deficiency [52]. In our study, we observed that the mRNA expression levels of $R S P O 2$ and its receptors are relatively high in brain samples compared to that in GBM cell lines (Fig. 1a). The protein Atlas data in human cerebral cortex illustrates that RSPO2 is mostly expressed at the neurophil area that forms a synaptically dense region containing mostly unmyelinated axons, dendrites and glial cell processes with a relatively low number of cell bodies in the brain (https://www.proteinatlas.org). Noticeably, RSPO2 is almost undetectable in glial cells, which are the cell of origin of glioma. Thus, compared to the normal glial cells, RSPO2 is highly expressed in GBM cell lines. Given the well-established role of $\mathrm{Wnt} / \beta$-catenin signaling in neural stem cell function and brain development, the regulatory effects of Rspos in neural stem cells warrant further investigation.

\section{Conclusions}

Altogether, the present study has demonstrated that Rspo 2 potentiates Wnt/ $\beta$-catenin signaling in GBM. In addition, we have shown that not only the baseline level of Wnt signaling, but also the functional response and adaptability to contextual Wnt ligands is the determinant for GBM stemness and should be pursued as GSC readout. The findings of this study reveal an undefined role of Rspos in the development of GBM, and shed new light into the underlying molecular mechanisms contributing to GSC regulation, which may provide grounds for the development of therapeutic targets for GBM.

\section{Additional files}

Additional file 1: Figure S1. Potentiation effect of Rspo2 in U87 cells. A-B, U87 cells were pre-treated in serum-free medium for 24 hours, then cultured in serum-free medium containing different WNT ligands for another 24 hours. Rspo 2 shows potentiation effect of Wnt3A induced $\beta$-catenin targets (A) as well as RSPO2-LGRs (B). Blk indicates U87 cells cultured in 
$0.1 \%$ DMSO. (C) Schematic illustration of 7TGP vector. Figure S2. The effect of Wnt3A and/or Rspos on stem cell markers in U87 and U251 cells. A, mRNA expression levels of stem cell markers in U87 cells in respond to Wnt3Aand/or Rspo2. Blk indicates U87 cells cultured in 0.1\% DMSO. B, Wnt $3 \mathrm{~A}$ and Rspo2 do not affect CD133 expression in U251 cells. U251 cells were pre-treated in serum-free medium for 24 hours, then cultured in serum-free medium containing different Wht ligands for another 24 hours. The cells were stained with CD133-APC antibody and analyzed for CD133 positivity by flow cytometry. Blk indicates U251 cells cultured in $0.1 \%$ DMSO. Figure S3. Establishment and Characterization of U251 and U87 GSCs. A, Flow cytometry analysis of U87 and U251 GSC-like cells. B, 5000 U251 cells or GSC-like cells were seeded in GSC medium for 10 days, sphere formation was evaluated for numbers and diameters. Quantification analysis of data is expressed as the Mean \pm SD from three independent experiments. C, 200 U251 cells or GSC-like cells were used for holoclone assay, where U251 GSCs show an enhanced holoclone formation ability than normal glioma cells. D-E, U87 GSCs show upregulated mRNA expression levels of $\beta$-catenin targets (D), as well as RSPO-LGR genes (E). Figure S4. Rspo2/Wnt3A prevents RA and growth factor deprivationinduced differentiation in GSCs. A, all-trans retinoic acid (10 $\mu \mathrm{M}$ RA) was used to induce differentiation in U87 GSCs for 24 hours with or without WNT ligands $(20 \mathrm{ng} / \mathrm{ml})$. Real-time PCR was used to determine the effect on differentiation. Results show that Rspo2/Wnt3A treatment rescues RA-induced U87 GSC differentiation. Blk indicates GSCs cultured in DMEM with $0.1 \%$ DMSO. B, U251 GSCs were cultured in GSC media, or GSC media without EGF and FGF, or GSC media without EGF and FGF but with Wnt3A and Rspo2 for 7 days. Phase image shows the morphology of spheres. C, real-time PCR shows that Rspo2/Wnt3A treatment abolishes the downregulation of $\beta$-catenin targets caused by growth factor deprivation. Blk indicates U251 GSCs cultured in GSC media with 0.1\% DMSO. Figure S5. Wnt ${ }^{\text {high }}$ and Wnt ${ }^{\text {low }}$ cell populations show different cellular behavior. A, Western blot analysis comparing the responsiveness of Wnt ${ }^{\text {high }}$ and Wnt ${ }^{\text {low }}$ cell populations. B, 3000 cells/well of U251 Wnt high and Wnt ${ }^{\text {low }}$ cells were pre-treated in serum-free medium for 24 hours, then cultured in serumfree medium containing different WNT ligands for another 4 days, and MTT assay was performed every 24 hours. C, Table shows serial dilution tumor inoculation assay using U251 Wnthigh and Wnt ${ }^{\text {tow }}$ cells.

Additional file 2: Table S1. Primer used for realtime PCR.

Additional file 3: Table S2. Antibodies used in Western blot.

\section{Authors' contributions}

$\mathrm{SL}$ : Experimental design and preformation, collection and/or assembly of data, data analysis and interpretation, manuscript writing. KPU, JZ, LLT, JH: Experimental preformation, collection and/or assembly of data, data analysis and interpretation. SPT: Discussion and interpretation of the data. XJ: Conception and experimental design, financial support, provision of study material, data analysis and interpretation, manuscript writing. All authors read and approved the final manuscript.

\section{Author details}

${ }^{1}$ Key Laboratory for Regenerative Medicine of the Ministry of Education of China, School of Biomedical Sciences, Faculty of Medicine, The Chinese University of Hong Kong, Hong Kong, SAR, People's Republic of China. ${ }^{2}$ School of Biomedical Sciences Core Laboratory, Shenzhen Research Institute, The Chinese University of Hong Kong, Shenzhen, People's Republic of China. ${ }^{3}$ Department of Oncology, Renji Hospital, School of Medicine, Shanghai Jiaotong University, Shanghai, China.

\section{Acknowledgements}

Not applicable.

\section{Competing interests}

The authors declare that they have no competing interests.

\section{Availability of data and materials}

All data generated or analyzed during this study are included in this published article and its supplementary information files.

\section{Consent for publication}

Not applicable.

\section{Ethics approval and consent to participate}

All animal experiments were conducted in accordance with the University Laboratory Animals Service Center's guidelines on animal experimentation with approval from the Animal Ethnics Committee of the University (Ref No. 16-002-MIS)

\section{Funding}

This work is supported by Hong Kong UGC/GRF Grants (466413, 14119516, 14165217) and Hong Kong Food and Health Bureau (01120056, 03140496).

\section{Publisher's Note}

Springer Nature remains neutral with regard to jurisdictional claims in published maps and institutional affiliations.

Received: 29 June 2018 Accepted: 5 October 2018 Published online: 11 October 2018

\section{References}

1. Gorlia T, van den Bent MJ, Hegi ME, Mirimanoff RO, Weller M, Cairncross JG, Eisenhauer E, Belanger K, Brandes AA, Allgeier A, et al. Nomograms for predicting survival of patients with newly diagnosed glioblastoma: prognostic factor analysis of EORTC and NCIC trial 26981-22981/CE.3. Lancet Oncol. 2008;9(1):29-38.

2. Huntly BJ, Gilliland DG. Leukaemia stem cells and the evolution of cancerstem-cell research. Nat Rev Cancer. 2005;5(4):311-21.

3. Al-Hajj M, Becker MW, Wicha M, Weissman I, Clarke MF. Therapeutic implications of cancer stem cells. Curr Opin Genet Dev. 2004;14(1):43-7.

4. Bao S, Wu Q, McLendon RE, Hao Y, Shi Q, Hjelmeland AB, Dewhirst MW, Bigner DD, Rich JN. Glioma stem cells promote radioresistance by preferential activation of the DNA damage response. Nature. 2006;444(7120):756-60.

5. Singh SK, Clarke ID, Terasaki M, Bonn VE, Hawkins C, Squire J, Dirks PB. Identification of a cancer stem cell in human brain tumors. Cancer Res. 2003;63(18):5821-8.

6. Yuan X, Curtin J, Xiong Y, Liu G, Waschsmann-Hogiu S, Farkas DL, Black KL, Yu JS. Isolation of cancer stem cells from adult glioblastoma multiforme. Oncogene. 2004;23(58):9392-400.

7. Trepant AL, Bouchart C, Rorive S, Sauvage S, Decaestecker C, Demetter P, Salmon I. Identification of OLIG2 as the most specific glioblastoma stem. cell marker starting from comparative analysis of data from similar DNA chip microarray platforms. Tumour Biol. 2015;36(3):1943-53.

8. Son MJ, Woolard K, Nam DH, Lee J, Fine HA. SSEA-1 is an enrichment marker for tumor-initiating cells in human glioblastoma. Cell Stem Cell. 2009;4(5):440-52.

9. Jin $\mathrm{X}$, Jin $\mathrm{X}$, Jung JE, Beck $\mathrm{S}$, Kim H. Cell surface Nestin is a biomarker for glioma stem cells. Biochem Biophys Res Commun. 2013;433(4):496-501.

10. Bradshaw A, Wickremsekera A, Tan ST, Peng L, Davis PF, Itinteang T. Cancer stem cell hierarchy in glioblastoma multiforme. Front Surg. 2016;3:21.

11. Choy W, Nagasawa DT, Trang A, Thill K, Spasic M, Yang I. CD133 as a marker for regulation and potential for targeted therapies in glioblastoma multiforme. Neurosurg Clin N Am. 2012;23(3):391-405.

12. Nusse R, Varmus HE. Many tumors induced by the mouse mammary tumor virus contain a provirus integrated in the same region of the host genome. Cell. 1982;31(1):99-109.

13. Schepers A, Clevers H. Wnt signaling, stem cells, and cancer of the gastrointestinal tract. Cold Spring Harb Perspect Biol. 2012;4(4):a007989.

14. Clevers H, Nusse R. Wnt/beta-catenin signaling and disease. Cell. 2012;149(6):1192-205.

15. Gurney A, Axelrod F, Bond CJ, Cain J, Chartier C, Donigan L, Fischer M, Chaudhari A, Ji M, Kapoun AM, et al. Wnt pathway inhibition via the targeting of Frizzled receptors results in decreased growth and tumorigenicity of human tumors. Proc Natl Acad Sci USA. 2012;109(29):11717-22.

16. Lee HJ, Bao J, Miller A, Zhang C, Wu J, Baday YC, Guibao C, Li L, Wu $\mathrm{D}$, Zheng JJ. Structure-based discovery of novel small molecule wnt 
signaling inhibitors by targeting the cysteine-rich domain of frizzled. J Biol Chem. 2015;290(51):30596-606.

17. Baarsma HA, Konigshoff M, Gosens R. The WNT signaling pathway from ligand secretion to gene transcription: molecular mechanisms and pharmacological targets. Pharmacol Ther. 2013;138(1):66-83.

18. Madan B, Virshup DM. Targeting Wnts at the source-new mechanisms, new biomarkers, new drugs. Mol Cancer Ther. 2015;14(5):1087-94.

19. Niehrs C. The complex world of WNT receptor signalling. Nat Rev Mol Cell Biol. 2012;13(12):767-79

20. Kahlert UD, Suwala AK, Koch K, Natsumeda M, Orr BA, Hayashi M, Maciaczyk J, Eberhart CG. Pharmacologic Wnt inhibition reduces proliferation, survival, and clonogenicity of glioblastoma cells. J Neuropathol Exp Neurol. 2015;74(9):889-900.

21. Bowman A, Nusse R. Location, location, location: FoxM1 mediates beta-catenin nuclear translocation and promotes glioma tumorigenesis. Cancer Cell. 2011;20(4):415-6.

22. Rossi M, Magnoni L, Miracco C, Mori E, Tosi P, Pirtoli L, Tini P, Oliveri G, Cosci E, Bakker A. beta-catenin and Gli1 are prognostic markers in glioblastoma. Cancer Biol Ther. 2011;1 1(8):753-61.

23. Lee Y, Lee JK, Ahn SH, Lee J, Nam DH. WNT signaling in glioblastoma and therapeutic opportunities. Lab Invest. 2016;96(2):137-50.

24. Reya T, Clevers H. Wnt signalling in stem cells and cancer. Nature. 2005;434(7035):843-50

25. de Lau WB, Snel B, Clevers HC. The R-spondin protein family. Genome Biol. 2012;13(3):242.

26. de Lau W, Peng WC, Gros P, Clevers H. The R-spondin/Lgr5/Rnf43 module: regulator of Wnt signal strength. Genes Dev. 2014;28(4):305-16.

27. Clevers H, Loh KM, Nusse R. Stem cell signaling: an integral program for tissue renewal and regeneration: Wnt signaling and stem cell control. Science. 2014;346(6205):1248012.

28. Carmon KS, Gong X, Lin Q, Thomas A, Liu Q. R-spondins function as ligands of the orphan receptors LGR4 and LGR5 to regulate Wnt/betacatenin signaling. Proc Natl Acad Sci USA. 2011;108(28):11452-7.

29. Hao HX, Xie Y, Zhang Y, Charlat O, Oster E, Avello M, Lei H, Mickanin C, Liu D, Ruffner $\mathrm{H}$, et al. ZNRF3 promotes Wnt receptor turnover in an R-spondin-sensitive manner. Nature. 2012;485(7397):195-200.

30. Koo BK, Spit M, Jordens I, Low TY, Stange DE, van de Wetering M, van Es $\mathrm{JH}$, Mohammed S, Heck AJ, Maurice MM, et al. Tumour suppressor RNF43 is a stem-cell E3 ligase that induces endocytosis of Wnt receptors. Nature. 2012;488(7413):665-9.

31. Theodorou V, Kimm MA, Boer M, Wessels L, Theelen W, Jonkers J, Hilkens J. MMTV insertional mutagenesis identifies genes, gene families and pathways involved in mammary cancer. Nat Genet. 2007;39(6):759-69.

32. Seshagiri S, Stawiski EW, Durinck S, Modrusan Z, Storm EE, Conboy CB, Chaudhuri S, Guan Y, Janakiraman V, Jaiswal BS, et al. Recurrent R-spondin fusions in colon cancer. Nature. 2012;488(7413):660-4.

33. Shinmura K, Kahyo T, Kato H, Igarashi H, Matsuura S, Nakamura S, Kurachi $\mathrm{K}$, Nakamura T, Ogawa H, Funai K, et al. RSPO fusion transcripts in colorectal cancer in Japanese population. Mol Biol Rep. 2014;41 (8):5375-84.

34. IImer M, Boiles AR, Regel I, Yokoi K, Michalski CW, Wistuba II, Rodriguez J, Alt E, Vykoukal J. RSPO2 enhances canonical Wnt signaling to confer stemness-associated traits to susceptible pancreatic cancer cells. Cancer Res. 2015;75(9):1883-96.

35. Storm EE, Durinck S, de Sousae Melo F, Tremayne J, Kljavin N, Tan C, Ye X, Chiu C, Pham T, Hongo JA, et al. Targeting PTPRK-RSPO3 colon tumours promotes differentiation and loss of stem-cell function. Nature. 2016;529(7584):97-100
36. Chartier C, Raval J, Axelrod F, Bond C, Cain J, Dee-Hoskins C, Ma S, Fischer MM, Shah J, Wei J, et al. Therapeutic targeting of tumor-derived R-Spondin attenuates beta-catenin signaling and tumorigenesis in multiple cancer types. Cancer Res. 2016;76(3):713-23.

37. Wu C, Qiu S, Lu L, Zou J, Li WF, Wang O, Zhao H, Wang H, Tang J, Chen L, et al. RSPO2-LGR5 signaling has tumour-suppressive activity in colorectal cancer. Nat Commun. 2014;5:3149.

38. Zhou X, Geng L, Wang D, Yi H, Talmon G, Wang J. R-Spondin1/LGR5 Activates TGFbeta signaling and suppresses colon cancer metastasis. Cancer Res. 2017;7(23):6589-602.

39. Berezovsky AD, Poisson LM, Cherba D, Webb CP, Transou AD, Lemke NW Hong X, Hasselbach LA, Irtenkauf SM, Mikkelsen T, et al. Sox2 promotes malignancy in glioblastoma by regulating plasticity and astrocytic differentiation. Neoplasia. 2014;16(3):193-206.

40. Zhang $\mathrm{S}$, Xie R, Wan F, Ye F, Guo D, Lei T. Identification of U251 glioma stem cells and their heterogeneous stem-like phenotypes. Oncol Lett. 2013;6(6):1649-55.

41. Auffinger B, Tobias AL, Han Y, Lee G, Guo D, Dey M, Lesniak MS, Ahmed AU. Conversion of differentiated cancer cells into cancer stem-like cells in a glioblastoma model after primary chemotherapy. Cell Death Differ. 2014;21(7):1119-31.

42. Yan KS, Janda CY, Chang J, Zheng GXY, Larkin KA, Luca VC, Chia LA, Mah AT, Han A, Terry JM, et al. Non-equivalence of Wnt and R-spondin ligands during Lgr5+ intestinal stem-cell self-renewal. Nature. 2017;545(7653):238-42.

43. de Sousa EMF, Vermeulen L. Wnt signaling in cancer stem cell biology. Cancers. 2016;8(7):E60.

44. Farin HF, Jordens I, Mosa MH, Basak O, Korving J, Tauriello DV, de Punder K, Angers S, Peters PJ, Maurice MM, et al. Visualization of a short-range Wnt gradient in the intestinal stem-cell niche. Nature. 2016;530(7590):340-3.

45. Kirchner T, Brabletz T. Patterning and nuclear beta-catenin expression in the colonic adenoma-carcinoma sequence Analogies with embryonic gastrulation. Am J Pathol. 2000;157(4):1113-21.

46. Henry C, Quadir A, Hawkins NJ, Jary E, Llamosas E, Kumar D, Daniels B, Ward RL, Ford CE. Expression of the novel Wnt receptor ROR2 is increased in breast cancer and may regulate both beta-catenin dependent and independent Wnt signalling. J Cancer Res Clin Oncol. 2015;141(2):243-54.

47. Brabletz T, Jung A, Hermann K, Gunther K, Hohenberger W, Kirchner T. Nuclear overexpression of the oncoprotein beta-catenin in colorectal cancer is localized predominantly at the invasion front. Pathol Res Pract. 1998;194(10):701-4

48. Cai C, Yu QC, Jiang W, Liu W, Song W, Yu H, Zhang L, Yang Y, Zeng YA. R-spondin 1 is a novel hormone mediator for mammary stem cell selfrenewal. Genes Dev. 2014;28(20):2205-18.

49. Knight MN, Hankenson KD. R-spondins: novel matricellular regulators of the skeleton. Mat Biol. 2014;37:157-61.

50. Bell SM, Schreiner CM, Wert SE, Mucenski ML, Scott WJ, Whitsett JA. $R$-spondin 2 is required for normal laryngeal-tracheal, lung and limb morphogenesis. Development. 2008;135(6):1049-58.

51. Yamada W, Nagao K, Horikoshi K, Fujikura A, Ikeda E, Inagaki Y, Kakitani M, Tomizuka K, Miyazaki H, Suda T, et al. Craniofacial malformation in R-spondin2 knockout mice. Biochem Biophys Res Commun. 2009;381(3):453-8.

52. Nam JS, Park E, Turcotte TJ, Palencia S, Zhan X, Lee J, Yun K, Funk WD, Yoon JK. Mouse R-spondin2 is required for apical ectodermal ridge maintenance in the hindlimb. Dev Biol. 2007;311(1):124-35. 